\title{
Inhibition of p38MAPK potentiates mesenchymal stem cell therapy against myocardial infarction injury in rats
}

\author{
ZHILING ZHANG ${ }^{1,2}$, SHULAN ZHOU ${ }^{2}$, ZHILIANG MEI $^{2}$ and MING ZHANG ${ }^{2}$ \\ ${ }^{1}$ Medical School of Nanchang University; ${ }^{2}$ The Second Department of Cardiovascular Medicine, \\ Jiangxi Provincial People's Hospital, Nanchang, Jiangxi 330006, P.R. China
}

Received June 7, 2016; Accepted April 11, 2017

DOI: $10.3892 / \mathrm{mmr} .2017 .6973$

\begin{abstract}
The present study aimed to investigate the protective effect of mesenchymal stem cell (MSC) therapy in combination with p38 mitogen-activated protein (MAPK) inhibition against myocardial infarction (MI) injury in rats, and to elucidate the underlying mechanisms. An MI model was established by ligation of the anterior descending branch of the left coronary artery. The rats were divided into four groups: MSC transplantation, p38MAPK inhibitor (SB203580), MSC + SB203580 and model control group. HE staining and a TUNEL assay were performed to evaluate pathological changes and apoptosis. The expression levels of p38MAPK and transforming growth factor $\beta$-activated kinase 1 (TAK1) were determined using reverse transcription-polymerase chain reaction and western blot analyses. As shown by HE staining, classical morphological changes, including irregular cell arrangement and inflammatory infiltration were observed in the model rats, whereas MSC therapy or injection of the p38MAPK inhibitor ameliorated these pathological changes. Of note, the combined application of MSCs with the p38MAPK inhibitor exerted additive effects. The TUNEL assay showed that the combined application of MSCs with p38MAPK inhibitor also led to potentiation of effects, compared with either MSCs therapy or p38MAPK inhibitor injection alone. Mechanistically, the combined application of MSCs with p38MAPK inhibitor decreased the expression levels of TAK1 and p38MAPK at the mRNA and protein levels. In conclusion, p38MAPK inhibition potentiated the protective effects of MSCs therapy against MI in rats.
\end{abstract}

Correspondence to: Dr Ming Zhang, The Second Department of Cardiovascular Medicine, Jiangxi Provincial People's Hospital, 92 Aiguo Road, Nanchang, Jiangxi 330006, P.R. China

E-mail: zhangming7819@sina.com

Key words: p38 mitogen-activated protein kinase, mesenchymal stem cells, myocardial infarction

\section{Introduction}

Myocardial infarction (MI) is caused by occlusion of the coronary artery, and is pathologically characterized by severe necrosis of cardiomyocytes due to persistent ischemia. MI is now a common disease affecting human health and coronary atherosclerosis is the major contributing factor for MI (1). In addition, hypertension, diabetes and blood lipid abnormalities can also contribute to MI, and smoking and alcohol abuse may be important inducers of blood abnormalities (2). Investigations using molecular methods have also suggested that the accumulation of reactive oxygen species stress, upregulation of adhesive molecules, and increases of serum homocysteine, blood fibrinogen and coagulation, may lead to abnormalities of blood lipids, resulting in MI (3).

Coronary atherosclerosis is elicited by a variety of endogenous and exogenous factors through different intracellular signaling pathways (4). MSC transplantation can promote angiogenesis in the infarct area to ameliorate cardiac function in MI (5). However, the clinical application of MSC transplantation is restricted by low survival rates of MSCs following transplantation (6). Several signaling pathways are abnormally regulated in the process of MI, including transforming growth factor- $\beta$ (TGF- $\beta$ )/small mothers against decapentaplegic, Janus kinase-signal transducers and activators of transcription, p38 mitogen-activated protein kinases (p38MAPK), phosphoinositide-3-kinase (PI3K)/Akt-endothelial nitric oxide synthase, PI3K/protein kinase C and Wnt (7-9). Among these signaling pathways, p38MAPK is involved in regulating the inflammatory process. Tumor necrosis factor (TNF)- $\alpha$ and interleukin (IL)-6 are classical pro-inflammatory factors, which are modulated by the p38MAPK pathway (8). The p38MAPK pathway is also important in cell differentiation and development (10).

p38MAPK functions through phosphorylating nuclear transcription factors and regulating gene expression. The inhibition of p38MAPK has been reported to protect against MI (4). However, the effects of the inhibition of p38MAPK on MSC therapy in an MI model remain to be elucidated. In the present study, an MI model was established in rats by ligation of the left anterior descending branch of the coronary artery. The MI model was exposed to the combined application of p38MAPK inhibition and MSC transplantation. The aim was to provide novel evidence for MI therapy. 


\section{Materials and methods}

Isolation and culture of MSCs. Male Sprague-Dawley (SD) rats (4-week old; $250 \pm 50 \mathrm{~g}$ ) were obtained from the Shanghai Laboratory Animal Center (SLAC; Shanghai, China). All experiments were approved by the ethics committee of Jiangxi Provincial People's Hospital (Nanchang, China). MSCs were isolated as previously described, with minor modifications (11). Briefly, $50 \mathrm{SD}$ rats were housed at room temperature with a standard 12-h light/dark cycle and ad libitum access to food and water for at least 7 days, following which the rats were sacrificed by cervical dislocation and soaked in $75 \%$ alcohol for 2 min. The bilateral tibial and femoral shafts were isolated and washed twice in cold PBS. The tibia was cut from the middle, and washed in low-glucose DMEM (Gibco; Thermo Fisher Scientific, Inc., Waltham, MA, USA) containing $100 \mathrm{U} / \mathrm{ml}$ heparin without serum. According to the Percoll lymphocyte separating method (cat. no. 17-0891-01; Pharmacia; GE Healthcare Life Sciences, Piscataway, NJ, USA), the suspension was centrifuged at a $500 \mathrm{~g}$ density gradient for $25 \mathrm{~min}$ at room temperature and the mononuclear cell layer was collected. The cells $\left(5 \times 10^{3} / \mathrm{ml}\right)$ were resuspended in low-glucose DMEM with 15\% FBS (GE Healthcare Life Sciences), $100 \mathrm{U} / \mathrm{ml}$ penicillin and streptomycin at $37^{\circ} \mathrm{C}$ and $5 \% \mathrm{CO}_{2}$. The cells were distinguished using flow cytometry with antibodies against CD34 (1:100; cat. no. ab23830, Abcam, Shanghai, China), CD44 (1:100; cat. no. ab46793, Abcam) and CD105 (1:100; cat. no. ab53318; Abcam). The cell growth curve is shown in Fig. 1.

MI model establishment. The specific pathogen-free rats (weight, $250 \pm 50 \mathrm{~g}$ ) were purchased from the SLAC and raised in an animal room for at least 7 days prior to experiments. The acute MI model was established by ligating the anterior descending branch of the coronary artery. Following anesthesia via intraperitoneal injection of $40 \mathrm{mg} / \mathrm{kg}$ pentobarbital with $0.1 \mathrm{mg} / \mathrm{kg}$ atropine, the rats were fixed on the bench in the supine position. A non-invasive method was used to insert a tube (18G) into the mouth in order to expose the throat. Positive pressure ventilation was applied using a ventilator to assist with animal breathing. The tidal volume was set at 9-12 ml and the breath rate was set at 1:1.5 with a frequency of $60 / \mathrm{min}$. Following exposure of the heart, the anterior descending branch of the left coronary artery was identified and ligated using 5-0 silk. The ST elevation was determined upon ECG and the tissues downstream of the coronary artery ligation were white or purple in color. Expansion of the left atrial appendage was performed to determine successful modeling of the MI model. The MI rats were then randomly divided into four groups: MSC therapy group, P38MAPK inhibition group, MSC therapy with p38MAPK inhibition group and saline control group. Subsequently, MSCs $\left(1.5 \times 10^{6} / 100 \mu 1\right)$ and/or SB203580 $(0.2 \mathrm{mg} / \mathrm{kg})$ were injected twice each week for a consecutive 4-week period. HE staining and a TUNEL assay were then performed to determine the pathological changes and apoptosis.

Reverse transcription-quantitative polymerase chain reaction (RT- $q P C R)$ analysis. The heart tissues were collected for RT-qPCR analysis. Total mRNA was isolated according to the manufacturer's protocol of the kit (cat. no. 74134; Qiagen, Inc., Valencia, CA, USA). The concentration and purity of the total RNA were determined using a Nanodrop 2000 spectrophotometer (Thermo Fisher Scientific, Inc.). Subsequently, the mRNAs underwent RT-qPCR combined with SYBR staining to amplify the expression (cat. no. RR430A; Takara Biotechnology Co., Ltd., Dalian, China). The primers were synthesized by Shanghai Sangon Biotech Co., Ltd. (Shanghai, China). The primers were as follows: p38MAPK, forward 5 '-tcgagaccgtttcagtccat-3' and reverse 5'-ccacggaccaaatatcca ct-3'; transforming growth factor $\beta$-activated kinase 1 (TAK1), forward 5'-ccatcccaatggcgtatcttac-3' and reverse 5'-tcatcctgg tccaattctgcaa-3'; GAPDH, forward 5'-acttgaagggtggagccaaa-3' and reverse 5 '-ccaggaaatgagcttgaca-3'. The $\mathrm{Cq}$ value for each gene was detected and the expression levels of target genes were calculated using the $2^{-\Delta \Delta \mathrm{Cq}}$ method (12). RT-qPCR was performed on a real-time PCR system (LightCycler 96; Roche Diagnostics, Basel, Switzerland) with the following procedure: Initial denaturation at $95^{\circ} \mathrm{C}$ for $5 \mathrm{~min}$, denaturation at $95^{\circ} \mathrm{C}$ for $30 \mathrm{sec}$, annealing at $60^{\circ} \mathrm{C}$ for $13 \mathrm{sec}$ and extension at $72^{\circ} \mathrm{C}$ for $30 \mathrm{sec}$, for 37 cycles. The reaction sample consisted of $10 \mu \mathrm{l}$ 2X SYBR Fast qPCR mix, $0.8 \mu 1$ forward/reverse primers $(10 \mathrm{mM}), 0.4 \mu \mathrm{l}$ 50X ROX Reference Dye II and $2 \mu \mathrm{l}$ cDNA template.

Western blot analysis. Myocardial tissues were collected for protein isolation (ReadyPrep; GE Healthcare Life Sciences). The protein concentrations were determined using a BCA protein assay kit (Thermo Fisher Scientific, Inc.). $25 \mu \mathrm{g}$ proteins from each group were obtained for sodium dodecyl sulfate polyacrylamide gel electrophoresis (12\%) and transferred onto nitrocellulose membranes for western blot analysis. The membranes were incubated with the following primary antibodies: p38MAPK (1:2,000; cat. no. sc-17852-R, Santa Cruz Biotechnology, Inc., Dallas, TX, USA), TAK1 (1:2,000; cat. no. sc-7967, Santa Cruz Biotechnology, Inc.) and actin (1:3,000; cat. no. sc-58673, Santa Cruz Biotechnology, Inc.). Following incubation with the primary antibodies at $4^{\circ} \mathrm{C}$ overnight, the nitrocellulose membranes were washed three times and incubated with secondary antibody (HRP-labeled goat anti-rabbit IgG; cat. no. A16104SAMPLE, Thermo Fisher Scientific, Inc.) at $4^{\circ} \mathrm{C}$ for $2 \mathrm{~h}$. The staining of the blots was enhanced using an ECL kit (Thermo Fisher Scientific, Inc.). The densities of the blots were quantified using Quantity One software (v4.62; Bio-Rad Laboratories, Inc., Hercules, CA, USA).

Statistical analyses. Data are presented as the mean \pm standard error of the mean. Statistical analyses were performed using one-way analysis of variance with GraphPad Prism 6.0 (GraphPad Software, Inc., La Jolla, CA, USA). P $<0.05$ was considered to indicate a statistically significant difference.

\section{Results}

Inhibition of p38MAPK potentiates the protective effect of MSC therapy against acute MI injury. An acute MI model was produced using rats by ligating the anterior descending branch of the coronary artery. The modeling process is shown in Fig. 2A. During the present study, four rats died due to 

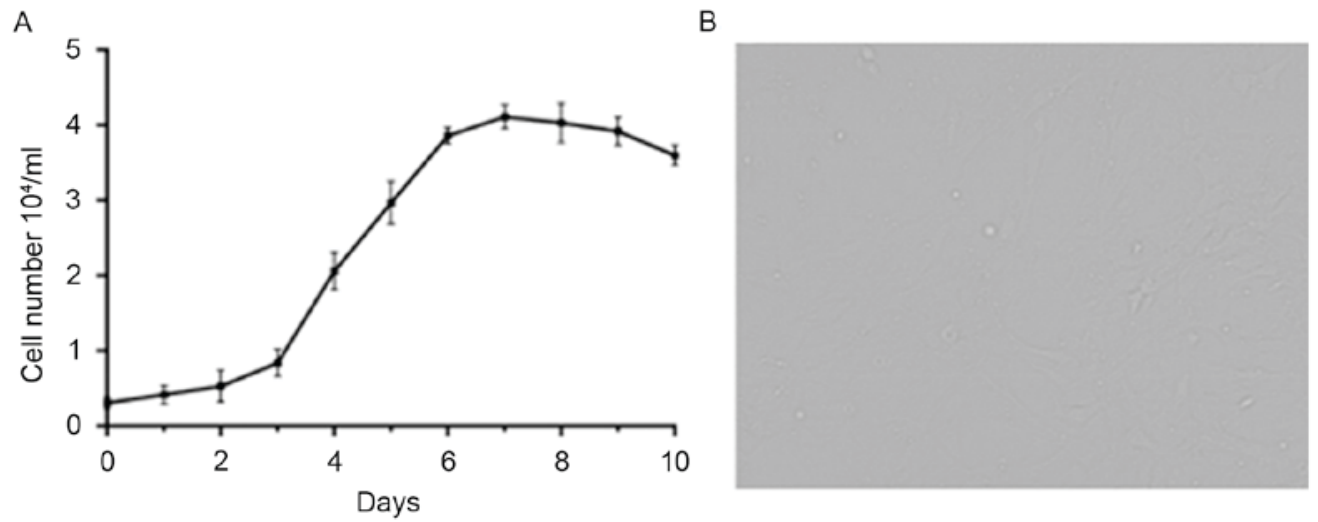

Figure 1. Cell growth. (A) Growth curve and (B) morphology of mesenchymal stem cells. Magnification, x200.
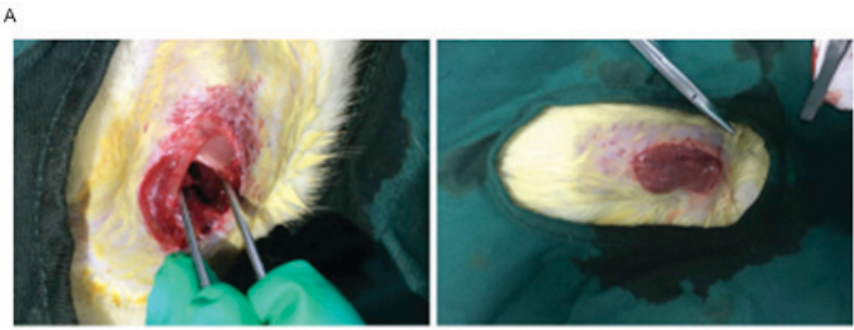

B

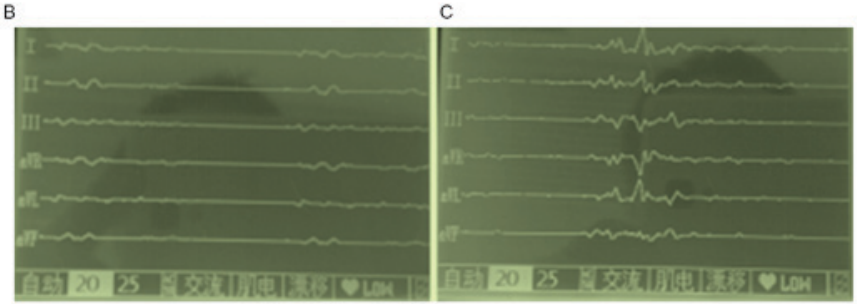

Figure 2. (A) MI surgery and ECG recordings of the (B) control and (C) MI model. MI, myocardial infarction.
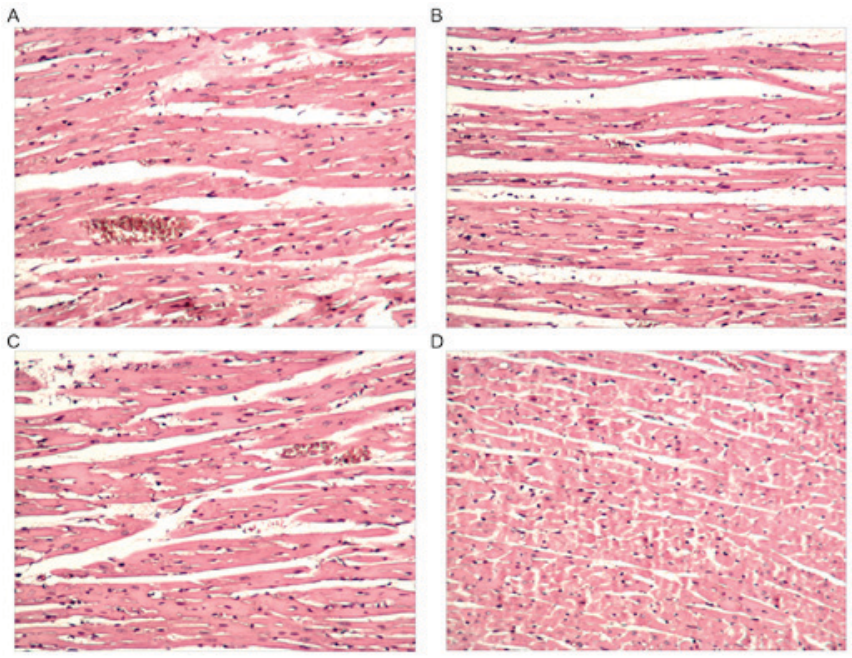

Figure 3. Pathological changes in heart tissues, determined using HE staining. (A) Control group; (B) MSC group; (C) SB203508 group; (D) MSC + SB203508 group. MSC, mesenchymal stem cell.

infection-induced heart failure. The ECG examination showed that the model rats exhibited acute anterior ST elevation
A

B
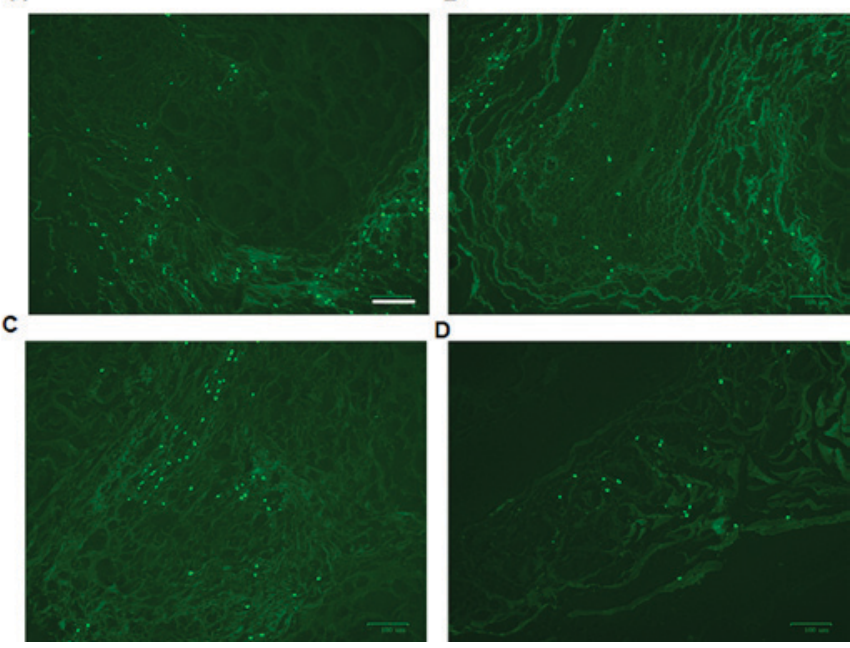

Figure 4. Detection of apoptosis using a TUNEL assay. (A) Control group; (B) MSC group; (C) SB203508 group; (D) MSC + SB203508 group. MSC, mesenchymal stem cell.

(Fig. 2B and C). At 4 weeks post-treatment, the heart tissues were collected for HE staining. As shown in Fig. 3A, the cells in the model control group were loosely arranged with numerous bubbles, inflammatory cell infiltration and tissue injury. Following treatment with MSCs or SB203580, the cells were tightly arranged with few bubbles (Fig. 3B and C). The combined application of MSCs with SB203580 exhibited optimal effects (Fig. 3D). A TUNEL assay was also performed to detect apoptosis. Apoptotic cells in the control group were apparent (Fig. 4A). Following treatment with MSCs or SB203580, the number of apoptotic cells was significantly decreased (Fig. 4B and C). Consistent with the HE staining, the combined application of MSCs with SB203580 (Fig. 4D) showed optimal effects in decreasing apoptosis.

Inhibition of p38MAPK and MSC therapy functions through the p38MAPK-TAK1 pathway. mRNA and proteins were isolated to examine the underlying mechanisms. The expression levels of p38MAPK and TAK1 were determined. Compared with the control group, MSC therapy or SB203580 injection significantly decreased the expression of p38MAPK 
A

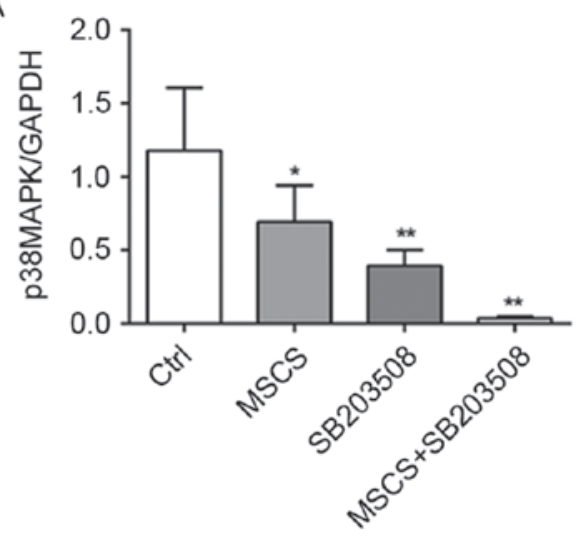

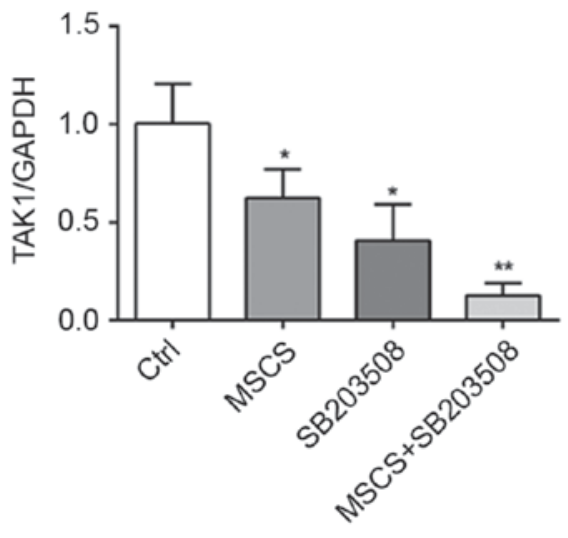

B

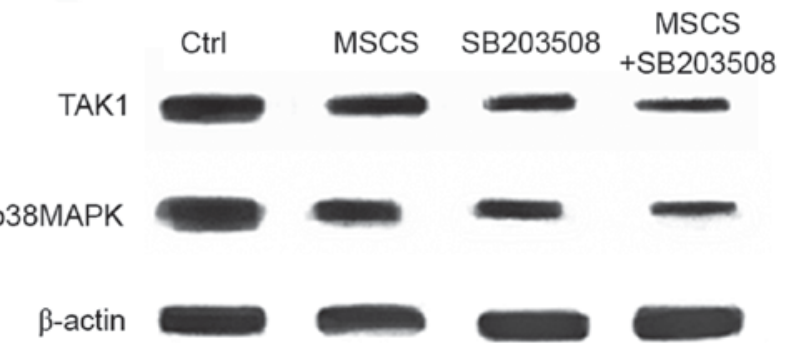

Figure 5. Effects of MSCs and SB203508 on the expression of p38MAPK and TAK1. (A) mRNA expression levels of p38MAPK and TAK1. (B) Protein expression levels of p38MAPK and TAK1. ${ }^{*} \mathrm{P}<0.05$ and ${ }^{* *} \mathrm{P}<0.01$, vs. Ctrl. MSCs, mesenchymal stem cells; MAPK, mitogen-activated protein kinase; TAK1, Transforming growth factor $\beta$-activated kinase 1 ; Ctrl, control.

at the mRNA (Fig. 5A) and protein (Fig. 5B) levels. In addition, the combined application of MSC therapy with SB203580 led to a further decrease in the expression of p38MAPK. The expression of TAK1 was also detected, which is upstream of the p38MAPK signaling pathway in the different groups. A consistent trend was observed in the groups as shown for p38MAPK (Fig. 5A and B).

\section{Discussion}

The MAPK family is a family of serine/threonine protein kinases, which can transfer signal transduction from the extracellular environment to the nucleus through the conservative three-cascade reaction (MAPKKK-MAPKK-MAPK) (13). There are four classical MAPK signaling pathways, including the extracellular signal-regulated kinase (ERK) signaling pathway, c-Jun N-terminal kinase/stress-activated protein kinase, p38 MAPK signaling pathway and the ERK5/big MAPK 1 signaling pathway. These signaling pathways have functions in several biological processes, including regulating cell migration, apoptosis, differentiation and proliferation (14). In the present study, it was found that the p38MAPK signaling pathway was critical in potentiating MSC therapy in MI injury.

The p38MAPK signaling pathway is one of the MAPK pathways, which can be activated by stress, cytokine stimulation, insulin and growth factor stimulation. In normal immune and inflammatory reactiona, the p38MAPK signaling pathway is activated to phosphorylate transcription factors and regulate gene expression, being involved in a variety of intracellular biological activities (15). Upstream of the p38MAPK signal transduction pathway, TAK1 regulates the phosphorylation of p38MAPK through the activation of TGF- $\beta$. Therefore, the expression or activation of TAK1 is an important indicator of the activity of the TGF- $\beta$-TAK1-p38MAPK signaling pathway. Several studies have suggested that the p38MAPK pathway is activated in the MI model $(5,16)$ and p38MAPK has become a therapeutic target for heart diseases $(17,18)$. However, other studies have demonstrated the pro-survival function of p38MAPK in MI $(19,20)$. In the present study, it was demonstrated that the inhibition of p38MAPK alone ameliorated the injury induced by MI. The expression of p38MAPK was also decreased following treatment with p38MAPK inhibitor. The inhibition of p38MAPK potentiated the protective effect of MSC therapy in MI.

Acute coronary artery occlusion causes blood flow interruption and leads to necrosis of heart tissue. Following MI, inflammatory cell infiltration, and serum levels of IL-6 and TNF- $\alpha$ are upregulated, suggesting that inflammation is closely associated with MI $(21,22)$. In the present study, ligation of the left coronary anterior descending branch was performed to produce MI, and the MI model was confirmed by ST elevation and pathological changes. This animal model is widely used to examine the mechanisms and enable screening of therapeutic agents. In the present study, the MI model was treated with p38MAPK inhibitor and/or MSCs twice each week for 4 weeks. MSCs are a type of non-hematopoietic stem cell found in bone marrow, which has the potential to differentiate into osteoblasts, fibroblasts, reticular cells, fat cells and endothelial cells. MSCs are characterized by their abundance, biodegradability and regeneration (22,23). Previously, MSC therapy has been used in the treatment of several diseases, including acute MI (24). In the present study, a Percoll lymphocyte separating system was applied to obtain MSCs. This separation system has the advantage of obtaining high purity 
MSCs. In the present study, the MI rats were randomly divided into four groups. Following MSC therapy or injection with the p38MAPK inhibitor, the pathological changes observed in the heart tissue were ameliorated. In addition, the combined application of MSCs with the p38MAPK inhibitor showed additive effects. The ameliorative effects were reflected by a decrease in inflammation and morphological changes. The results of the TUNEL assay also indicated that that number of apoptotic cells following MI was significantly attenuated by MSC therapy or the inhibition of p38MAPK.

In conclusion, the present study evaluated the effects of inhibiting p38MAPK on MSC therapy against MI injury. Although the inhibition of p38MAPK or MSC therapy alone exerted mild protective effects, their combined application had a superior effect. These findings provide novel implications for the clinical application of MSC therapy for MI.

\section{References}

1. Pitts R, Daugherty SL, Tang F, Jones P, Ho PM, Tsai TT, Spertus J and Maddox TM: Optimal secondary prevention medication use in acute myocardial infarction patients with nonobstructive coronary artery disease is modified by management strategy: Insights from the TRIUMPH Registry. Clin Cardiol: Apr 7, 2017 (Epub ahead of print) doi: 10.1002/clc.22686.

2. Shibutani H, Akita Y, Yutaka K, Yamamoto S, Matsui Y, Yoshinaga M, Karakawa M and Mori Y: Acute myocardial infarction with 'wrap around' right coronary artery mimicking Takotsubo cardiomyopathy: A case report. BMC Cardiovasc Disord 16: 71, 2016.

3. Sun HJ, Zhao MX, Ren XS, Liu TY, Chen Q, Li YH, Kang YM, Wang JJ and Zhu GQ: Salusin- $\beta$ promotes vascular smooth muscle cell migration and intimal hyperplasia after vascular injury via ROS/NFאB/MMP-9 pathway. Antioxid Redox Signal 24: 1045-1057, 2016.

4. Ma SF, Luo Y, Ding YJ, Chen Y, Pu SX, Wu HJ, Wang ZF, Tao BB, Wang WW and Zhu YC: Hydrogen sulfide targets the Cys320/Cys529 motif in Kv4.2 to inhibit the Ito potassium channels in cardiomyocytes and regularizes fatal arrhythmia in myocardial infarction. Antioxid Redox Signal 23: 129-147, 2015.

5. Li G, Qian W and Zhao C: Analyzing the anti-ischemia-reperfusion injury effects of ginsenoside Rb1 mediated through the inhibition of p38 $\alpha$ MAPK. Can J Physiol Pharmacol 94: 97-103, 2016.

6. Wang F, Zhou H, Du Z, Chen X, Zhu F, Wang Z, Zhang Y, Lin L, Qian M, Zhang X, et al: Cytoprotective effect of melatonin against hypoxia/serum deprivation-induced cell death of bone marrow mesenchymal stem cells in vitro. Eur J Pharmacol 748: $157-165,2015$.

7. Wei N, Zhang C, He H, Wang T, Liu Z, Liu G, Sun Z, Zhou Z, Bai $C$ and Yuan D: Protective effect of saponins extract from Panax japonicus on myocardial infarction: Involvement of $\mathrm{NF}-\kappa \mathrm{B}$, Sirt1 and mitogen-activated protein kinase signalling pathways and inhibition of inflammation. J Pharm Pharmacol 66: 1641-1651, 2014.
8. Mehta PK and Griendling KK: Angiotensin II cell signaling: Physiological and pathological effects in the cardiovascular system. Am J Physiol Cell Physiol 292: C82-C97, 2007.

9. Xiao H, Xu YN, Luo H, Chen Y, Zhang YY, Tao L, Jiang Y and Shen XC: OMT inhibited TGF- $\beta 1$-induced cardiac fibroblast proliferation via down-regulating p38MAPK phosphorylation in vitro. Zhongguo Zhong Yao Za Zhi 40: 2168-2173, 2015 (In Chinese).

10. Blanc A, Pandey NR and Srivastava AK: Synchronous activation of ERK 1/2, p38mapk and PKB/Akt signaling by $\mathrm{H} 2 \mathrm{O} 2$ in vascular smooth muscle cells: Potential involvement in vascular disease (review). Int J Mol Med 11: 229-234, 2003.

11. Bourzac C, Smith LC, Vincent P, Beauchamp G, Lavoie JP and Laverty S: Isolation of equine bone marrow-derived mesenchymal stem cells: A comparison between three protocols. Equine Vet J 42: 519-527, 2010.

12. Zhu G, Li J, He L, Wang X and Hong X: MPTP-induced changes in hippocampal synaptic plasticity and memory are prevented by memantine through the BDNF-TrkB pathway. Br J Pharmacol 172: 2354-2368, 2015.

13. Osaki LH and Gama P: MAPKs and signal transduction in the control of gastrointestinal epithelial cell proliferation and differentiation. Int J Mol Sci 14: 10143-10161, 2013.

14. del Barco Barrantes I and Nebreda AR: Roles of p38 MAPKs in invasion and metastasis. Biochem Soc Trans 40: 79-84, 2012.

15. Miloso M, Scuteri A, Foudah D and Tredici G: MAPKs as mediators of cell fate determination: An approach to neurodegenerative diseases. Curr Med Chem 15: 538-548, 2008.

16. Arabacilar $\mathrm{P}$ and Marber M: The case for inhibiting p38 mitogen-activated protein kinase in heart failure. Front Pharmacol 6: 102, 2015.

17. O'Donoghue ML, Glaser R, Aylward PE, Cavender MA, Crisp A, Fox KA, Laws I, Lopez-Sendon JL, Steg PG, Theroux P, et al: Rationale and design of the LosmApimod To Inhibit p38 MAP kinase as a TherapeUtic target and moDify outcomes after an acute coronary syndromE trial. Am Heart J 169: 622-630.e6, 2015.

18. Wang M, Li Z, Zhang X, Xie X, Zhang Y, Wang X and Hou Y: Rosuvastatin attenuates atrial structural remodelling in rats with myocardial infarction through the inhibition of the p38 MAPK signalling pathway. Heart Lung Circ 24: 386-394, 2015.

19. Mitra A, Ray A, Datta R, Sengupta S and Sarkar S: Cardioprotective role of P38 MAPK during myocardial infarction via parallel activation of $\alpha$-crystallin B and Nrf2. J Cell Physiol 229: 1272-1282, 2014.

20. Li C, He J, Gao Y, Xing Y, Hou J and Tian J: Preventive effect of total flavones of Choerospondias axillaries on ischemia/reperfusion-induced myocardial infarction-related MAPK signaling pathway. Cardiovasc Toxicol 14: 145-152, 2014.

21. Huang M, Yang D, Xiang M and Wang J: Role of interleukin-6 in regulation of immune responses to remodeling after myocardial infarction. Heart Fail Rev 20: 25-38, 2015.

22. Somasuntharam I, Yehl K, Carroll SL, Maxwell JT, Martinez MD, Che PL, Brown ME, Salaita K and Davis ME: Knockdown of TNF- $\alpha$ by DNAzyme gold nanoparticles as an anti-inflammatory therapy for myocardial infarction. Biomaterials 83: 12-22, 2016.

23. Sekar D, Saravanan S, Karikalan K, Thirugnanasambantham K, Lalitha P and Islam VI: Role of microRNA 21 in mesenchymal stem cell (MSC) differentiation: A powerful biomarker in MSCs derived cells. Curr Pharm Biotechnol 16: 43-48, 2015.

24. Hu X, Chen P, Wu Y, Wang K, Xu Y, Chen H, Zhang L, Wu R, Webster KA, Yu H, et al: MiR-211/STAT5A signaling modulates migration of mesenchymal stem cells to improve its therapeutic efficacy. Stem Cells 34: 1846-1858, 2016. 